\title{
BEAM-LOADING COMPENSATION FOR SUPER B-FACTORIES *
}

\author{
D. Teytelman ${ }^{\dagger}$ \\ SLAC, Menlo Park, CA 94025, USA
}

\section{Abstract}

Super B-factory designs under consideration expect to reach luminosities in the $10^{35}-10^{36}$ range. The dramatic luminosity increase relative to the existing $\mathrm{B}$-factories is achieved, in part, by raising the beam currents stored in the electron and positron rings. For such machines to succeed it is necessary to consider in the RF system design not only the gap voltage and beam power, but also the beam loading effects. The main effects are the synchronous phase transients due to the uneven ring filling patterns and the longitudinal coupled-bunch instabilities driven by the fundamental impedance of the RF cavities. A systematic approach to predicting such effects and for optimizing the RF system design will be presented. Existing as well as promising new techniques for reducing the effects of heavy beam loading will be described and illustrated with examples from the PEP-II and the KEKB.

\section{INTRODUCTION}

Two super B-factory designs under development aim to increase the peak luminosity from currently achieved $9 \cdot 10^{33}-15 \cdot 10^{33}$ level to the $10^{35}-10^{36}$ range $[1,2]$. The dramatic luminosity increase requires a ten-fold increase in the beam currents stored in electron and positron rings. High beam currents in large circumference storage rings cause two significant beam loading effects: fast-growing longitudinal coupled-bunch instabilities excited by the detuned fundamental mode and the synchronous bunch phase transient due to the fill pattern unevenness.

Significant attention to the beam loading was already paid in the design of the existing B-factories with the two machines selecting radically different solutions [3, 4]. Projected beam current increases for the super B-factories amplify the significance of the beam loading making it the defining element in the design of the RF system.

In this paper the concept of beam loading in a storage ring is introduced and several important relationships between the cavity parameters and beam current are presented. Next, two beam loading effects are described and the existing methods for reducing these harmful effects are presented. From a general description of RF design and feedback control methods that alleviate the beam loading issues a "cookbook" procedure for designing super Bfactory RF system is then developed. The procedure is il-

\footnotetext{
* Work supported by U.S. Department of Energy contract DE-AC0276SF00515

†dim@slac.stanford.edu
}

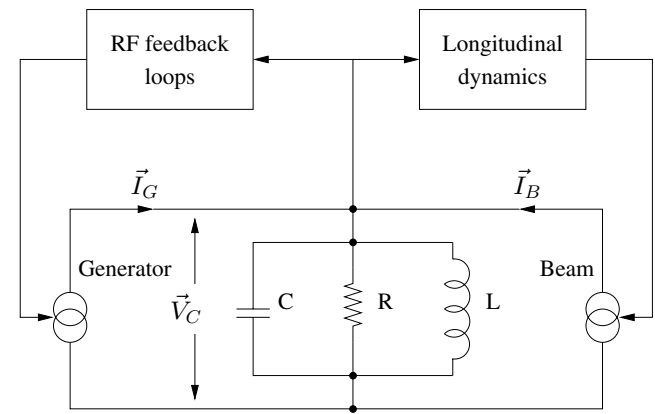

Figure 1: An RLC model of the fundamental mode of the RF cavity where $R$ is the loaded cavity shunt impedance.

lustrated with a design example from the SuperPEP.

\section{BEAM-CAVITY INTERACTION}

\section{Lumped-element model of the RF cavity}

Let's start our discussion of the beam loading from considering an RLC model of the accelerating or fundamental mode of the RF cavity. Such a model is illustrated in Fig. 1. The fundamental mode is driven by two input currents, the generator current $\vec{I}_{G}$ and the beam current component at the RF frequency $\vec{I}_{B}$. The cavity voltage $\vec{V}_{C}$ is then defined by the vector sum of the input currents and the impedance of the fundamental mode. In turn the cavity voltage influences the beam current via the longitudinal dynamics and the generator currents through the RF feedback loops.

In Fig. 2 a vector diagram describing the relationship be-

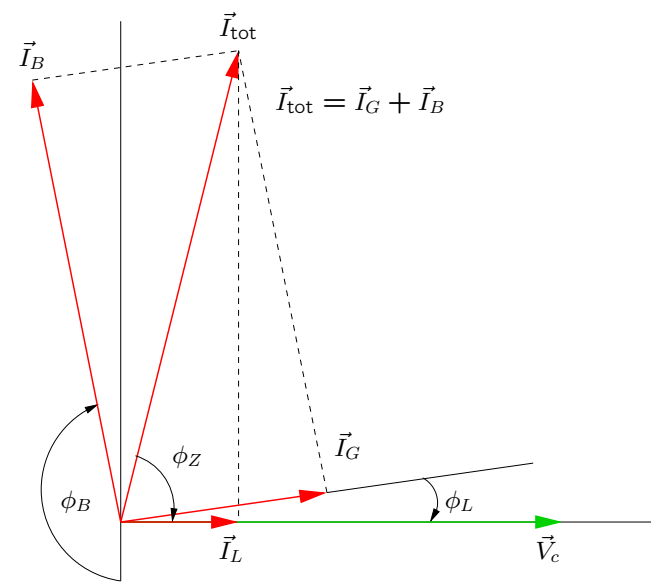

Figure 2: Vector diagram of the cavity currents and voltage 
tween the generator and beam currents and the cavity voltage is shown. For the short bunches typically encountered in B-factories the beam current component at $\omega_{\mathrm{rf}}$ is twice the DC beam current $I_{0}$. Synchronous phase angle $\phi_{B}$ is determined by the total RF voltage $V_{T}$ and the energy loss per turn $U_{0}$ as $\phi_{B}=\pi-\arcsin \left(U_{0} / V_{T}\right)$. The angle $\phi_{Z}$ between the total input current $\vec{I}_{\text {tot }}$ and the voltage $\vec{V}_{c}$ is the cavity impedance phase angle $\angle Z_{c}\left(i \omega_{\mathrm{rf}}\right)$. The current $I_{L}$ is loss current in the model shunt impedance $R$ which includes the effects of coupling to the generator output. For the steady-state conditions from the geometric considerations we have

$$
I_{G}=\frac{I_{L}+I_{B} \sin \phi_{B}}{\cos \phi_{L}}
$$

Note that the minimum generator current is achieved at the zero loading angle $\phi_{L}$. For efficient use of the generator power the cavity is detuned as a function of the beam current to maintain $\phi_{L}=0$. From the vector diagram in Fig. 2 we get the following expression for the cavity phase angle

$$
\tan \phi_{Z}=\tan \phi_{L}+\frac{I_{B}}{I_{L}}\left(\tan \phi_{L} \sin \phi_{B}+\cos \phi_{B}\right)
$$

Cavity resonance detuning from the $\mathrm{RF}$ frequency is related to the cavity phase angle as $\omega_{D}=\omega_{r}-\omega_{\mathrm{rf}}=\sigma \tan \phi_{Z}$ where $\sigma$ is the cavity damping time $\omega_{r} /\left(2 Q_{L}\right)$. If the loading angle is zero, from Eq. 1 we obtain the following expression for the detuning frequency

$$
\omega_{D}=\frac{\omega_{r}}{2 Q_{L}} \frac{I_{B}}{I_{L}} \cos \phi_{B}=\frac{\omega_{r} I_{B}}{2 V_{c}} \frac{R}{Q} \cos \phi_{B}
$$

\section{Beam loading}

When the beam current is small relative to the generator current - light beam loading - the cavity voltage is mostly defined by the generator current. High beam current starts to affect strongly the cavity voltage thus creating a strong interaction between the RF system and the beam - high beam loading condition. This interaction can be considered as a "feedback loop": beam current source is affected by the cavity voltage, while that voltage depends on the beam current.

Another way to look at beam loading is to consider Eq. 2. Cavity detuning increases linearly with beam current and in a large circumference high-current storage rings one encounters a condition where $\omega_{D}$ is comparable to the ring revolution frequency $\omega_{0}$ or even exceeds it. In this case the fundamental impedance interacts strongly with the beam current components at the revolution frequency (uneven filling patterns) as well as the components at the synchrotron sidebands (longitudinal coupled-bunch instabilities).
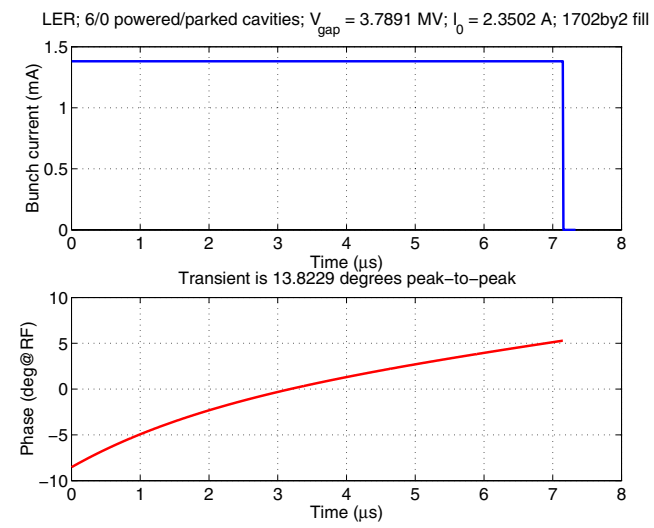

Figure 3: Computed synchronous phase transient in PEP-II LER for a gap of $88 \mathrm{RF}$ buckets or $2.52 \%$

\section{Synchronous phase transients}

Let's first consider the effect of the uneven ring filling pattern. Such unevenness is an amplitude modulation of the beam current input to our cavity model. The modulation is periodic from turn to turn therefore in the frequency domain the beam signal has power at the revolution harmonics. These components interact with the detuned cavity impedance producing both amplitude and phase modulations of the cavity voltage which, in turn, influences the longitudinal parameters of the individual bunches. Affected parameters include synchrotron frequency, bunch length, and the synchronous position.

The most significant feature of a B-factory filling pattern is a relatively long gap for the abort kicker risetime and ion clearing. Such a pattern in time domain generates an envelope of the revolution frequency harmonics around the RF frequency with the amplitude and the width of the envelope related to the length of the gap as follows

$$
I\left(\omega_{\mathrm{rf}}+n \omega_{0}\right)=\left|2 I_{b} \frac{\sin \pi n N_{g} / h}{\sin \pi n / h}\right|
$$

where $I(\omega)$ is the magnitude of the beam current component at frequency $\omega, I_{b}$ is the per bunch current, $N_{g}$ is the length of the gap and $h$ is the harmonic number. For the low values of $n$ consistent with the span of the fundamental impedance Eq. 3 can be approximated by $2 I_{b} N_{g}$.

In the simplest form a product of the fill pattern spectrum and the cavity impedance will give us the expected cavity voltage modulation. From the modulation one can then compute the variations in the synchronous position and other bunch parameters. Note, however, that cavity voltage modulation leads to the phase modulation of the beam current vector changing the impedance excitation spectrum. This is the feedback loop effect mentioned earlier which leads to significant errors when phase transients are large. In order to accurately compute the transient we use the small-signal model by F. Pedersen [5]. Figure 3 illustrates a synchronous phase transient in PEP-II LER for a $2.52 \%$ gap. At the $2.35 \mathrm{~A}$ beam current the transient amplitude 


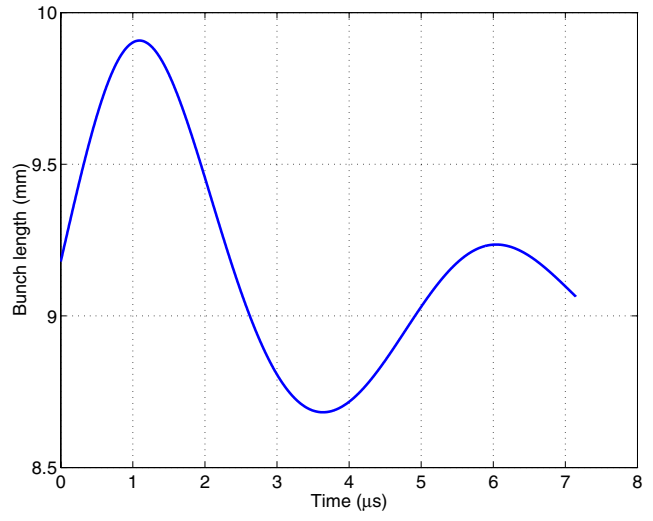

Figure 4: Bunch length variation in the LER for a $2.52 \%$ gap in the filling pattern

reaches $13.8^{\circ}$ peak-to-peak. Modulation of the cavity voltage also leads to variation of the bunch length along the train illustrated in Fig. 4. The time structure in the plot is related to the resonant center frequency of the RF cavities at $202 \mathrm{kHz}$ (1.5 revolution harmonics) detuning.

There are several ways of combating the synchronous phase transients. From the previous discussion it is clear that reducing the length of the abort gap leads to a nearly linear reduction in the amplitude of the transient. For $\omega_{D}<$ $\omega_{0}-2 \sigma$ cavity impedance at $\omega_{\mathrm{rf}}-\omega_{0}$ can be written as

$$
\left|Z_{c}\left(\omega_{\mathrm{rf}}-\omega_{0}\right)\right| \approx \frac{R}{Q} \frac{\omega_{\mathrm{rf}}}{2\left(\omega_{0}-\omega_{D}\right)}
$$

Several intuitively reasonable conclusions can be drawn from this relation. One way to reduce the impedance thus reducing the phase transient it to lower $R / Q$ which is equivalent to increasing the cavity stored energy. Secondly, keeping cavity detuning low helps in the reduction. Based on Eq. 2 we see that increased cavity stored energy (low $R / Q$ ) and increased cavity voltage also help lower the detuning. Note that the impedance is also proportional to the number of RF cavities.

Let us now consider an active approach to lessening the phase transient. If one were to apply appropriate modulation to $\vec{I}_{G}$ a perfect cancellation of the fill pattern gap term could be achieved. Such a cancellation, however, extracts a significant power penalty requiring peak generator current to increase by a factor of 2-3 [4, page 195]. A more promising approach is described in [6] where a question is posed: what reduction in the amplitude of the transient can be achieved with only the phase modulation of $\vec{I}_{G}$ which places no additional demands on the available RF power. Analysis of the PEP-II HER examples shows that a $12^{\circ}$ transient is reduced by a factor of 4 to $3^{\circ}$.

\section{Longitudinal coupled-bunch instabilities}

As we have seen the fundamental impedance interacts strongly with the beam current components at the revolution harmonics. Another important interaction takes place at the synchrotron sidebands of the revolution harmonics leading to the longitudinal coupled-bunch instabilities. The eigenvalue shifts of the even fill eigenmodes (EFEMs) are given by [7]

$$
\begin{aligned}
\lambda_{l} & =\frac{\pi \alpha e f_{\mathrm{rf}}^{2} I_{0}}{E_{0} h \omega_{s}} Z^{\| \mathrm{eff}}\left(l \omega_{0}+\omega_{s}\right) \\
Z^{\| \mathrm{eff}}(\omega) & =\frac{1}{\omega_{\mathrm{rf}}} \sum_{p=-\infty}^{\infty}\left(p \omega_{\mathrm{rf}}+\omega\right) Z^{\|}\left(p \omega_{\mathrm{rf}}+\omega\right)
\end{aligned}
$$

where $l$ is the eigenmode number, $\alpha$ is the momentum compaction, $E_{0}$ is the beam energy, and $\omega_{s}$ is the synchrotron frequency. If we consider only the fundamental mode of the RF cavities from Eqs. 5-6 it follows that

$$
\lambda_{l} \propto Z_{c}\left(\omega_{\mathrm{rf}}+l \omega_{0}+\omega_{s}\right)-Z_{c}^{*}\left(\omega_{\mathrm{rf}}-l \omega_{0}-\omega_{s}\right)
$$

where $Z^{*}$ denotes a complex conjugate operation.

Without the beam the cavity is tuned on resonance and the impedance at the upper synchrotron sideband of a revolution harmonic below the RF is nearly equal to the impedance at the lower synchrotron sideband of the opposite harmonic. As the cavity is detuned to compensate for the beam loading the asymmetry increases reaching a maximum when $\omega_{D}$ is near $\omega_{0}-\omega_{s}$. As a result the eigenvalue of mode -1 is shifted to very fast growth rates.

Many modern storage rings operate above the coupledbunch instability threshold and use a longitudinal bunchby-bunch feedback system to control the beam [8]. To understand why the situation with the cavity fundamental impedance requires different treatment we will consider the limitations of such feedback systems.

Longitudinal bunch-by-bunch feedback control is typically achieved by measuring individual bunch displacements from the synchronous phase, processing the signals to compute a corrective kick, and applying such kick via a power amplifier and kicker. In a conventional system the kick for a given bunch is applied at least one turn after its displacement has been measured. In practice the delay is significantly higher since the kick signal needs 90 degrees phase shift relative to the position oscillation. Generating such a shift requires observations of the bunch position over multiple turns comprising a significant fraction of the synchrotron oscillation period. These requirements place a lower bound on the feedback controller group delay which, in turn, limits achievable loop gains and damping rates. The limitation is illustrated in Fig 5 where a root locus of the closed loop system is shown. Within the plot the locus (red) starts at the open-loop eigenvalue (marked by a green "x") for zero feedback gain. As the loop gain increases the feedback acts to shift the eigenvalue to the left at first reducing the growth rate and then, after crossing the y-axis, achieving stability. Further gain increases initially improve damping, however the eigenvalue curve turns around near $-9 \mathrm{~ms}^{-1}$. In addition another closed-loop pole locus (blue) enters from the left and is driven towards instability. In 


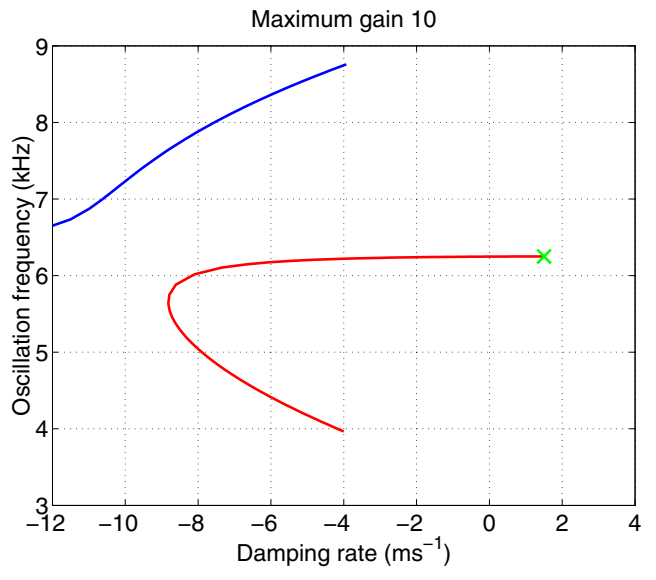

Figure 5: Root locus of a low group-delay longitudinal feedback controller in PEP-II HER acting on a eigenmode with $1.5 \mathrm{~ms}^{-1}$ growth rate

this illustration an overall distance between the open-loop eigenvalue and the maximum damping is on the order of $10.5 \mathrm{~ms}^{-1}$. The illustrated feedback loop is a low groupdelay woofer channel in PEP-II HER [9] which achieves $77 \mu$ s group delay - roughly half of the synchrotron oscillation period of $156 \mu \mathrm{s}$. Increasing modal growth rates will shift the overall locus plot to the right with the system running out of control margin at the growth rates near $10 \mathrm{~ms}^{-1}$. In practice a $50 \%$ margin is advisable for reliable control which then halves the supportable growth rates.

While feedback systems have other limitations such as kicker gain, available amplifier power, and input sensitivity, only the group-delay is a fundamental performance limit. This is then the limit for the RF system design.

Two approaches to reducing the instability growth rates induced by the fundamental impedance include impedance minimization [3] and active feedback [4]. Impedance minimization techniques are very similar to the methods of reducing the synchronous phase transient that were described earlier. The similarity is due to the fact that in case of phase transients we are minimizing the impedance at the revolution harmonic while in case of the instabilities to the first order the impedance at the upper synchrotron sideband of that harmonic is minimized.

Active feedback control of the fundamental-mode impedance has been successfully used in several large storage rings, most notably SPS [10] and PEP-II [11]. In a most general sense the system compares cavity probe signal to a fixed reference and applies correction via the generator to keep the error small. Then a change in the cavity voltage due to a beam current change is attenuated by the feedback leading to a reduced effective impedance. The feedback loop response is comprised of two channels: direct and comb loops. The direct loop is a prompt proportional feedback channel with no frequency shaping. The gain of this channel and the achievable impedance reduction is limited by the loop transport delay which includes klystron group delay, waveguide and probe cable delays as well as the LLRF processing. Note, however, that the beam-cavity interaction driving the coupled-bunch instabilities takes place in the vicinity of the synchrotron sidebands of the revolution harmonics near $\omega_{\mathrm{rf}}$. A second feedback loop - comb loop - trades bandwidth for gain and provides additional loop gain at the synchrotron sidebands. This loop uses a near one-turn delay with an equalizer so that the loop response is periodic at the revolution harmonics providing appropriately phased gain peaks at the synchrotron sidebands. In practice impedance reduction achieved by these feedback loops falls short of linear model predictions due to the small-signal amplitude saturation of the klystron. From Fig. 2 it is clear that rejection of beam phase oscillations requires mostly amplitude modulation of the klystron. In a nominal operating regime a klystron is $85-90 \%$ saturated with the small-signal gain attenuated by $10-12 \mathrm{~dB}$. This attenuation effectively reduces the loop gain and cannot be compensated by the feedback gain change since the phase modulation path is not attenuated. As a result in PEPII we have observed residual impedance roughly 5 times larger than the linear model prediction.

\section{RF PARAMETER SELECTION PROCEDURE}

Based on the recommendations developed in the previous two sections a "cookbook" procedure that minimizes both important beam loading effects can be formulated.

\section{Assumptions}

In the parameter selection procedure several assumptions will be made. First, only superconducting (SC) cavities are considered since the minimum number of cavities, as we will see, is determined by the beam power requirement. Even though in the existing B-factories both conventional and energy-storage normal-conducting cavities are used, in a new design these options would lead to a larger number of cavities than if the SC cavities are employed. Next I will assume that the synchronous phase angle $\phi_{B}$ is close to $\pi$, so that $\cos \phi_{B} \approx 1$ - a reasonable assumption given the large overvoltage factors being considered. We will assume that all HOM losses in the cavity occur above cut-off, that is the resonator is effectively single-mode below the cut-off frequency. Finally, the RF parameter selection procedure assumes a pre-determined bunch length requirement and a significant flexibility in the selection of the momentum compaction factor.

\section{Methodology}

In this design procedure the first step is to determine the cavity design limits on the gap voltage $V_{c}$ and the power coupled into the cavity by the generator $P_{g}$. We will want to operate all cavities in the ring at these limits since the detuning is proportional to $1 / V_{c}$ and the number of cavities scales with $1 / P_{g}$. From the cavity design we get the above 
cut-off HOM loss factor $k_{s}$ which gives us per cavity HOM power loss $P_{\mathrm{HOM}}=I_{0}^{2} k_{s} / f_{\mathrm{rf}}$. The difference $P_{b}^{c}=P_{g}-$ $P_{\text {HOM }}$ is the power supplied to the beam by each cavity. Next we determine total beam power requirement $P_{t}$ based on the synchrotron radiation, resistive wall losses as well as HOM losses elsewhere in the vacuum chamber. These two quantities determine the required number of cavities $N_{c}=\left\lceil P_{t} / P_{b}^{c}\right\rceil$. Having determined the number of cavities we right away get the total gap voltage $V_{G}=N_{c} V_{c}$. Next we use the equations for the synchrotron frequency and the bunch length [12]

$$
\begin{aligned}
\sigma_{z} & =\frac{\alpha c}{\omega_{s}} \delta_{E} \\
\omega_{s}^{2} & =\frac{\alpha e \omega_{\mathrm{rf}}}{E_{0} T_{0}} V_{G}
\end{aligned}
$$

where $\sigma_{z}$ is the bunch length, $\delta_{E}$ is the fractional energy spread, $T_{0}=2 \pi / \omega_{0}$ is the revolution time. Eqs. 8-9 can be rewritten to express momentum compaction as a function of other parameters, determined earlier

$$
\alpha=\frac{\omega_{\mathrm{rf}} e \sigma_{z}^{2}}{E_{0} T_{0} c^{2} \delta_{E}^{2}} V_{G}
$$

\section{SuperPEP example}

Let's demonstrate the above procedure on the SuperPEP LER ring at $3.5 \mathrm{GeV}$ nominal energy and $15.5 \mathrm{~A}$ beam current with design bunch length of $1.8 \mathrm{~mm}$ [2].

Currently in the SuperPEP RF system design there are three cavity options under consideration. All of these are $952 \mathrm{MHz}$ SC cavities with $P_{g}$ of $1 \mathrm{MW}$ and $V_{c}$ of $1.25 \mathrm{MV}$. The cavity options have different $R / Q: 30 \Omega, 12 \Omega$, and $5 \Omega$. In this example we will only consider the cavity with $R / Q=12 \Omega$. Overall beam power requirement is 20.12 MW. Cavity loss factor $k_{s}$ is $0.31 \mathrm{~V} / \mathrm{pC}$ which gives us HOM power loss of $79 \mathrm{~kW}$. Thus each cavity delivers $921 \mathrm{~kW}$ to the beam and we need 22 cavities. Total gap voltage is $27.5 \mathrm{MV}$. Using Eq. 10 we get $\alpha=3.6 \cdot 10^{-4}$. Synchrotron frequency for this configuration is $7.65 \mathrm{kHz}$.

Under the full beam loading the cavity detuning is $142 \mathrm{kHz}$ which exceeds the revolution frequency of $136 \mathrm{kHz}$. This necessitates LLRF feedback to reduce the impedance. Applying direct and comb loops as well as the effects of klystron saturation we get a growth rate of $4 \mathrm{~ms}^{-1}$. Linearizing the klystron [13] would reduce the residual rate to $0.8 \mathrm{~ms}^{-1}$. Both of these rates fall within the controllable range of the coupled-bunch feedback systems. It is interesting to note that a cavity with $R / Q$ of $5 \Omega$ reaches only $61 \mathrm{kHz}$ of detuning and can be operated without impedance-reduction feedback.

\section{SUMMARY}

High current storage rings must pay careful attention to the harmful beam loading effects. Longitudinal coupled-bunch instabilities due to the cavity fundamental impedance to large extent define the RF system design for a highly beam loaded large storage ring. Reducing the growth rates of such instabilities to a manageable level will most likely involve a combination of several methods: design stage impedance minimization through cavity and ring parameters and active LLRF feedback. An example presented here illustrates the RF parameter selection and design choices to achieve manageable instability growth rates at the very high stored beam currents.

\section{ACKNOWLEDGMENTS}

Author would like to thank P. McIntosh, A. Novokhatski, J. Seeman, M. Sullivan, and U. Wienands for productive discussions of SuperPEP RF and accelerator parameters.

\section{REFERENCES}

[1] S. Hashimoto et al., Letter of intent for KEK Super B Factory. KEK, 2004. KEK-REPORT-2004-4.

[2] J. Seeman, M. Sullivan, and U. Wienands, "Parameters of a Super-B-Factory design," these proceedings.

[3] K. Akai et al., "RF systems for the KEK B-factory," Nucl. Instrum. Meth., vol. A499, pp. 45-65, 2003.

[4] F. Pedersen, "RF cavity feedback," in $B$ factories: the state of the art in accelerators, detectors and physics: proceedings, pp. 192-207, SLAC, 1992.

[5] F. Pedersen, "Beam loading effects in the CERN PS booster," IEEE Trans. Nucl. Sci., vol. 22, pp. 1906-1909, 1975.

[6] P. B. Wilson, "Fundamental mode RF design in e+ e- storage ring factories," in Frontiers of Particle Beams: Factories with e+e-Rings: proceedings of a topical course, pp. 293 311, Springer-Verlag, 1994.

[7] S. Prabhakar et al., "Observation and modal analysis of coupled-bunch longitudinal instabilities via a digital feedback control system," Part. Accel., vol. 57, p. 175, 1997.

[8] J. D. Fox and E. Kikutani, "Bunch feedback systems and signal processing," in Beam Measurement: Proceedings, pp. 579-620, World Scientific, 1999.

[9] D. Teytelman, D. Van Winkle, and J. Fox, "Operating performance of the low group delay woofer channel in PEP-II," these proceedings.

[10] D. Boussard and G. Lambert, "Reduction of the apparent impedance of wide band accelerating cavities by RF feedback," IEEE Trans. Nucl. Sci., vol. 30, p. 2239, 1983.

[11] P. Corredoura, L. Sapozhnikov, and R. Tighe, "RF feedback development for the PEP-II B factory," in EPAC 94: proceedings, (River Edge, NJ, USA), pp. 1954-1956, World Scientific, 1994.

[12] M. Sands, "The physics of electron storage rings: An introduction," Tech. Rep. SLAC-R-121, SLAC, Stanford, CA, USA, 1970.

[13] J. Fox et al., "Klystron linearizer for use with 1 MW 476 MHz klystrons in PEP-II RF systems," these proceedings. 